\title{
A comparative study of visible light polymerized gelatin hydrogels for 3D culture of hepatic progenitor cells
}

\author{
Tanja Greene, ${ }^{1}$ Tsai-Yu Lin, ${ }^{1}$ Ourania M Andrisani, ${ }^{2}$ and Chien-Chi Lin ${ }^{1, *}$ \\ ${ }^{1}$ Department of Biomedical Engineering, Indiana University-Purdue University Indianapolis, \\ Indianapolis, IN, 46202, USA \\ ${ }^{2}$ Department of Basic Medical Sciences and Purdue Center for Cancer Research, Purdue \\ University, West Lafayette, IN, 47907, USA
}

*To whom correspondence should be made:

Prof. Chien-Chi Lin

Associate Professor of Biomedical Engineering

Indiana University-Purdue University Indianapolis

723 W. Michigan St. SL220K

Indianapolis, IN 46202

Phone: (317)274-0760

Email: lincc@iupui.edu

This is the author's manuscript of the article published in final edited form as:

Greene, T., Lin, T.-Y., Andrisani, O. M. and Lin, C.-C. (2016), Comparative study of visible light polymerized gelatin hydrogels for 3D culture of hepatic progenitor cells. J. Appl. Polym. Sci., 134, 44585.

http://dx.doi.org/10.1002/app.44585 


\begin{abstract}
Photopolymerization techniques have been widely used to create hydrogels for biomedical applications. Visible light-based photopolymerizations are commonly initiated by type II (i.e., non-cleavage type) photoinitiator in conjunction with a co-initiator. On the other hand, type I photoinitiators (i.e., cleavage type) are rarely compatible with visible light-based initiation due to their limited molar absorbability in the visible light wavelengths. Here, we report visible light initiated orthogonal photo-click crosslinking to fabricate gelatin-norbornene (GeINB) and poly(ethylene glycol)-tetra-thiol (PEG4SH) hydrogels using either cleavage-type (i.e., lithium acylphosphinate, LAP) or non-cleavage type photoinitiator (i.e., eosin-Y, EY) without the use of a co-initiator. Regardless of the initiator type, the step-growth gelatin-PEG hybrid hydrogels crosslinked and degraded similarly. While both systems exhibited similar cytocompatibility for hepatic progenitor HepaRG cells, gelation initiated by non-cleavage type initiator eosin-Y afforded slightly higher degree of hepatic gene expression.
\end{abstract}




\section{Introduction}

Hydrogels prepared from photopolymerizations have been used extensively in biomedical applications, including three dimensional (3D) cell culture for tissue engineering and regenerative medicine. Among the commonly used macromolecules for hydrogel fabrication, gelatin is a preferable option because of its inherent bioactivity and biocompatibility. While unmodified gelatin molecules do not form stable 3D networks at a physiological temperature, modified gelatin can be readily polymerized into covalent hydrogels that are not only more stable but have better tunability in material properties (e.g., stiffness). Gelatin can be easily modified with different polymerizable motifs, such as methacrylamide,$^{[1,2]}$ thiol, ${ }^{[3]}$ and norbornene. ${ }^{[4-6]}$ We have previously reported the modular crosslinking of gelatin-based hydrogels using gelatin-norbornene (GelNB) and poly(ethylene glycol)-tetra-thiol (PEG4SH). ${ }^{[4,5]}$ The crosslinking reaction could be rapidly initiated by cleavage-type photoinitiator lithium acylphosphinate (LAP) under low intensity of longwave ultraviolet light (UV) exposure $\left(5-10 \mathrm{~mW} / \mathrm{cm}^{2}, \lambda=365 \mathrm{~nm}\right)$. We demonstrated that the material properties of the resulting hydrogels could be modularly controlled due to the orthogonal thiol-norbornene crosslinking. Furthermore, these modular gelatin-based hydrogels could be employed for culturing human mesenchymal stems cells (hMSCs) ${ }^{[4]}$ and hepatocellular carcinoma cells $(\mathrm{HCC})^{[5]}$ in 3D. Nonetheless, the use of UV light to initiate gelation may not be ideal for some biomedical applications as the short wavelengths of UV light could induce cellular damage. Ongoing efforts of the field are to prepare biomimetic hydrogels from highly cytocompatible visible light initiated orthogonal photochemistry.

Visible light (wavelength: $400-700 \mathrm{~nm}$ ) is arguably a more cytocompatible light source than UV light for initiating the crosslinking of cell-laden hydrogels. The photoinitiators capable of being excited by visible light to induce hydrogel crosslinking include type II initiators eosin, ${ }^{[7]}$ rose bengal, ${ }^{[8]}$ and ruthenium, ${ }^{[9]}$ as well as type I initiator lithium acylphosphinate (LAP). ${ }^{[10]}$ While type I (cleavage type) photoinitiator by itself is sufficient in initiating photopolymerization, most of the type II photoinitiators (non-cleavage type) require a co-initiator to achieve reasonable gelation kinetics. We have shown that it is possible to initiate thiol- 
norbornene gelation using visible light and type II initiator eosin-Y without using a co-initiator. ${ }^{[7,}{ }^{11]}$ Specifically, the thiol moiety is deprotonated by visible light excited eosin-Y, thus forming thiyl radicals capable of reacting with the norbornene group to form orthogonal thiol-ether bond. This visible light mediated thiol-norbornene reaction has not been applied to the orthogonal crosslinking of gelatin-based hydrogels.

Photopolymerized hydrogels have been used for creating liver-mimetic tissue models for studying liver cell physiology and drug toxicity. Primary human hepatocytes $(\mathrm{PHH})$ are the gold standard for testing drug toxicity, metabolism, and viral infection in vitro. However, PHHs de-differentiate (i.e., loss of hepatocyte-specific functions and phenotype) rapidly during in vitro culture, either on two-dimensional (2D) surfaces or on 3D cell culture. Alternatively, hepatocellular carcinoma (HCC) derived hepatic cell lines (eg. HepG2, Huh7) could be used to study liver diseases. While convenient and readily available, these immortalized cell lines do not exhibit liver-specific characteristics, including the expression of some hepatic-specific genes and proteins. Recently, a bi-potent liver progenitor cell line HepaRG is being actively pursued as a potential cell type for studying liver regeneration, drug testing, and liver-specific viral infection (e.g., hepatitis B virus or HBV). ${ }^{12-}$ 15] Immature HepaRG cells are classified as bi-potent hepatoblasts that can be differentiated towards cholangiocytic (biliary) and hepatocytic cells. ${ }^{[14,16,17]}$ Differentiated HepaRG cells possess hepatocyte-like characteristics, and unlike isolated PHHs that are easily de-differentiated during in vitro culture, ${ }^{[18]}$ HepaRG cells exhibit a more stable phenotype during extended in vitro culture. ${ }^{[13,15,19]}$ Unfortunately, established protocols for differentiating HepaRG cells require the use of dimethyl sulfoxide (DMSO) at a cytotoxic concentration ( 2\%). ${ }^{[20]}$ Even though DMSO could induce hepatocyte differentiation in HepaRG cells, the reported differentiation efficiency varies greatly from batch to batch. ${ }^{[19]}$

Several research groups have explored the use of 3D culture methods to induce hepatic differentiation of HepaRG cells. For example, Higuchi et al. cultured HepaRG cell spheroids in suspension using media containing a low-acyl gellan gum polymer (FP001). ${ }^{[17]}$ The presence of FP001 polymer permitted the suspension of HepaRG cell spheroids in culture media for up to 28 days without the need for 
stirring. In addition, the levels of hepatocyte differentiation were higher than that in conventional 2D culture. While this culture method promoted the differentiation of HepaRG cells into more hepatocytes than cholangiocytes, this method still required the use of $1.7 \%$ DMSO. In view of the cytotoxic nature of DMSO, others have been experimenting with DMSO-free 3D cultures to induce hepatocytic differentiation in HepaRG cells. For instance, hydrogels comprised of either physically crosslinked nanofibrillar cellulose or covalently crosslinked hyaluronan modified gelatin were used to culture and differentiate HepaRG progenitor cells. ${ }^{[16]}$ While being cultured within a 3D matrix, HepaRG cells could still form clusters and expressed measureable levels of hepatocyte-specific genes (i.e., HNF4a, ALB, and CYP3A4). In another example, HepaRG cell spheroids were encapsulated in alginate microgels and maintained in stirred DMSO-free culture medium for up to 4 weeks. ${ }^{[19]}$ It was determined that cells maintained in 3D aggregates without DMSO showed similar levels of hepatocyte-specific transcription factors (i.e., HNF4 $\alpha$ and PXR) compared to cells cultured in 2D and treated with DMSO. Together, these studies demonstrated the possibility of inducing hepatic differentiation in HepaRG cells without using DMSO.

In this contribution, we report DMSO-free culture and potential hepatic differentiation of HepaRG cells in gelatin-based 3D hydrogels formed by visible light initiated thiol-norbornene photochemistry. Although others have reported the use of gelatin for 3D culture of hepatocytes, none have utilized orthogonally crosslinked thiol-norbornene gelatin-based hydrogels for culturing HepaRG cells. ${ }^{[16,21,}$ 22] Here, we systematically evaluated the effect of photoinitiator (type I or type II) on gelation efficiency, hydrogel properties (e.g., gel stiffness and proteolytic degradability), and cytocompatibility of gelatin-based hydrogels. In addition, we examined the influence of photoinitiator type on viability and potential hepatocytic differentiation of progenitor HepaRG cells in a DMSO-free 3D hydrogel culture environment. 


\section{Experimental Section}

\subsection{Materials}

Type A gelatin (Bloom 238-282) and 4-arm PEG-SH (M.W. 10 kDa) were purchased from Amresco and JenKem Technology USA, respectively. Collagenase (300 U/mg) was obtained from Worthington Biochemical. AlamarBlue ${ }^{\circledR}$ reagent and CYP450 assay kit were obtained from Thermo Scientific. Glutamax and Live/Dead staining kit for mammalian cells, penicillin and streptomycin were purchased from Life Technologies. DPBS and newborn calf serum (NCS) were acquired from Lonza and HyClone, respectively. William's Medium E, human insulin solution, hydrocortisone hemisuccinate, and all other chemicals were obtained from Sigma-Aldrich unless otherwise noted.

\subsection{Synthesis of GeINB}

GelNB synthesis was achieved through reacting gelatin with carbic anhydride as described previously with slight modification. ${ }^{[4]}$ Briefly, $10 \mathrm{wt} \%$ of gelatin was dissolved in $50^{\circ} \mathrm{C}$ DPBS, followed by the addition of carbic anhydride (20 wt/vol\%) to the solution. The $\mathrm{pH}$ value of the solution was adjusted to 7.5-8.0 using $2 \mathrm{~N}$ sodium hydroxide solution. After 72 hours of reaction, 3-fold volume of warm DPBS $\left(37^{\circ} \mathrm{C}\right)$ was added to the solution, and any undissolved carbic anhydride was removed by centrifugation. The GelNB solution was dialyzed (MWCO of dialysis membrane: $6-8 \mathrm{kDa}$ ) against $\mathrm{ddH}_{2} \mathrm{O}$ at $40{ }^{\circ} \mathrm{C}$ for 3 days. Finally, the GelNB solution was freeze-dried to obtain the dry product. The degree of norbornene substitution was quantified by fluoraldehyde assay using unmodified gelatin with known concentrations as standards. The degree of substitution was determined to be $\sim 2.2 \mathrm{mM}$ norbornene moieties per 1 wt $\%$ of GeINB. 


\subsection{Hydrogel Fabrication and Characterization}

Orthogonally crosslinked gelatin hydrogels were fabricated via step-growth photopolymerization using GeINB and crosslinker PEG4SH. Pre-polymer solutions containing desired amounts of GeINB, PEG4SH, and photoinitiator (LAP or EY) were exposed to a halogen cold light lamp (400 - $700 \mathrm{~nm}$, AmScope) for 5 minutes at $70 \mathrm{k}$ Lux (equivalent to $10 \mathrm{~mW} / \mathrm{cm}^{2}$ at $550 \mathrm{~nm}$ ). In some control experiments, polymerization was initiated via UV light exposure (365 $\mathrm{nm}, 5 \mathrm{~mW} / \mathrm{cm}^{2}$ for 5 minutes). To quantify gelation efficiency, pre-polymer solution (100 $\left.\mu \mathrm{L}\right)$ was loaded in the light cure cell of a Bohlin CVO 100 digital rheometer. ${ }^{[4]}$ In situ photorheometry was conducted using the same visible light source connected to a flexible light guide with the light switched on 10 seconds after initiating the time-sweep measurement $(25 \mathrm{~mm}$ parallel plate geometry, $10 \%$ strain, $1 \mathrm{~Hz}$ frequency, and a gap size of $90 \mu \mathrm{m})$. The time when storage or shear modulus (G') surpassed loss modulus (G") was labeled as the gel point. To obtain shear modulus of the hydrogels, gels were pre-formed by injecting aliquots of pre-polymer solutions between glass slides separated by $1 \mathrm{~mm}$ Teflon spacers. Disc-shaped hydrogels were prepared using an $8 \mathrm{~mm}$ diameter biopsy punch. Gel moduli were measured using an $8 \mathrm{~mm}$ parallel plate with a gap size of $750 \mu \mathrm{m}$. Oscillatory rheometry was performed in strain-sweep mode $(0.1 \%$ to $5 \%$ strain; $1 \mathrm{~Hz}$ frequency). Averaged G' values were determined from the linear portion of the modulus-strain curves.

\subsection{Proteolytic Degradation of GelNB Hydrogels}

After photopolymerization of hydrogels, the original mass of the gel $\left(M_{0}\right)$ was obtained gravimetrically. The gels were then placed in wells with $0.5 \mathrm{~mL}$ PBS containing collagenase type I (10 U/mL). At given time intervals, gels were removed from protease solution, blotted dry, and the mass recorded $\left(\mathrm{M}_{\mathrm{t}}\right)$. This process was repeated until no remnants of the gel could be seen. 
Results were presented as percent mass loss, which was defined as: $\%$ Mass loss $=100 \% \times$ $\left(\mathrm{M}_{0}-\mathrm{M}_{\mathrm{t}}\right) / \mathrm{M}_{0}$

\subsection{Cell Culture and Encapsulation}

HepaRG cells were cultured using conditions described previously. ${ }^{[12]}$ Briefly, cells were maintained in Williams Medium E supplemented with 10\% NCS, $100 \mu \mathrm{g} / \mathrm{mL}$ streptomycin, 100 $\mathrm{U} / \mathrm{ml}$ penicillin, $50 \mu \mathrm{M}$ hydrocortisone hemisuccinate, and $5 \mu \mathrm{g} / \mathrm{mL}$ human insulin. Cells were passaged every two weeks (between passage 13 to 20), with media refreshed twice a week. To encapsulate HepaRG cells, cells were trypsinized, counted, and suspended in DPBS to obtain a final cell density of $5 \times 10^{6}$ cells $/ \mathrm{mL}$ in all hydrogels. Both macromers (i.e., GelNB, PEG4SH) and photoinitiator (i.e., eosin-Y or LAP) were dissolved in PBS and sterilized by passing the solutions through $0.22 \mu \mathrm{m}$ syringe filters. Macromers, photoinitiator, and cells were mixed at desired concentrations with gentle pipetting. $20 \mu \mathrm{L}$ aliquots of precursor solutions were subjected to the same light irradiation conditions as described above. The cell-laden hydrogels were cultured in HepaRG growth media and maintained at $37^{\circ} \mathrm{C}$ in $5 \% \mathrm{CO}_{2}$. Culture media were refreshed twice a week.

\subsection{Characterizations of Cell Viability and Function}

To investigate cell viability within hydrogels, encapsulated HepaRG cells were stained with live/dead staining kit and imaged with a confocal microscope (Olympus Fluoview FV100 laser scanning microscope). Z-stack images (100 $\mu \mathrm{m}$ thick, $10 \mu \mathrm{m}$ per slice) from at least three random fields of a gel were captured for all conditions. To quantitatively evaluate cell viability, AlamarBlue ${ }^{\circledR}$ reagent was used following a previously developed protocol. ${ }^{[23]}$ CYP450 enzymatic activity was assayed using Luciferin-MultiCYP assay kit on days 14 and 28 postencapsulation. In brief, encapsulated cells were incubated in $300 \mu \mathrm{L}$ of HepaRG growth media containing Luciferin-MultiCYP $(100 \mu \mathrm{M})$ for 1 hour at $37^{\circ} \mathrm{C}$ in $5 \% \mathrm{CO}_{2}$. Next, $50 \mu \mathrm{L}$ of Luciferin- 
media from each sample was transferred to a 96-well opaque white luminometer plate containing $50 \mu \mathrm{L}$ luciferin detection reagent per well. Samples were allowed to incubate at room temperature for 20 minutes and a microplate reader (SynergyHT, BioTek) was utilized to detect the luminescence. To account for the variation in cell numbers from different gels, enzyme activity levels were normalized by the cell metabolic activity from the respective cell-laden hydrogel.

\subsection{RNA Isolation, Reverse Transcription, and Quantitative Real Time PCR}

On days 14 , and 28 , hydrogels containing encapsulated HepaRG cells were collected, placed in DNase/RNase-free microtubes, and flash frozen using liquid nitrogen. Samples were then stored at $-80^{\circ} \mathrm{C}$ until use. To extract RNA, frozen gels were homogenized in $600 \mu \mathrm{L}$ of lysis buffer (NucleoSpin RNA II kit, Clontech), followed by two additional freeze-thaw cycles in order to lyse cells. Lysates were cleared using NucleoSpin filters, followed by mixing with $600 \mu \mathrm{L}$ of RNase-free $70 \%$ ethanol. The mixtures were then passed through NucleoSpin RNA columns to allow RNA binding. RNA bound to the membranes of the columns was eluted using $30 \mu \mathrm{L}$ of DNase/RNase-free water and quantified by UV/vis spectrometry (NanoDrop 2000, Thermo Scientific).

Reverse transcription of isolated RNA (8-130 ng) into cDNA was achieved using PrimeScript RT reagent kit (Clontech). Gene expression level was assessed by quantitative real-time PCR on an Applied Biosystems 7500 fast real-time PCR machine and analyzed following a published protocol. ${ }^{[24]}$ In short, samples were run at $95^{\circ} \mathrm{C}$ for $30 \mathrm{~s}$, followed by 45 cycles of $95^{\circ} \mathrm{C}$ for $5 \mathrm{~s}, 55^{\circ} \mathrm{C}$ for $30 \mathrm{~s}$, and at $72^{\circ} \mathrm{C}$ for $30 \mathrm{~s}$. Within each experimental group, the detected levels of the genes of interest were normalized to the level of house-keeping gene GAPDH. Gene expression levels were calculated using $2^{-\Delta \Delta C T}$ method where the expression level of 2D culture was set as 1-fold after having normalized all other groups to the 2D undifferentiated groups for each respective day. Real-time PCR primer sequences (forward and 
reverse) are: GAPDH: 5'-GAAGGTGAAGGTCGGAGTC-3' and 5'GAAGATGGTGATGGGATTTC-3'; HNF4a: 5'-GCCTACCTCAAAGCCATCAT-3' and 5'GACCCTCCCAGCAGCATCTC-3'; NTCP (SLC10A1): 5'-GGGACATGAACCTCAGCATT-3' and 5'- CGTTTGGATTTGAGGACGAT-3'.

\subsection{Statistics}

All statistical analyses were conducted using GraphPad Prism 5 software. Experiments were conducted independently at least three times with each experimental or control group containing a minimum of three samples per group. Results were presented as Mean $\pm \mathrm{SD}$. Twoway ANOVA with Bonferroni post-test was performed to evaluate the statistical significance between the indicated groups and $p<0.05$ was considered statistically significant $\left({ }^{*}\right.$ denote $p<$ $0.05,{ }^{* *}$ denote $p<0.001,{ }^{\star * \star}$ denote $\left.p<0.0001\right)$.

\section{Results \& Discussion}

\subsection{Orthogonal Crosslinking of GelNB-PEG4SH Hydrogels}

We have previously shown that the crosslinking of gelatin-based hydrogels could be initiated via long wavelength UV light exposure $\left(365 \mathrm{~nm}, 5-10 \mathrm{~mW} / \mathrm{cm}^{2}\right)$ and with the use of cleavage type photoinitiator LAP at a relatively low concentration (i.e., $1 \mathrm{mM}){ }^{[4,5]}$ Although longwave UV light-based gelation was rapid and cytocompatible for many cells, concerns regarding UV light exposure prompted us to develop visible light-based orthogonal gel crosslinking methods. In this regard, we have reported that step-growth thiol-norbornene gel crosslinking could be achieved by eosin-Y initiated visible light photopolymerization using multiarm PEG-norbornene and bi-functional thiol moieties (e.g., dithiothreitol, DTT). ${ }^{[7]}$ Here, we sought to test whether orthogonal crosslinking of gelatin-based hydrogels could also be initiated and tuned using visible light exposure. First, 5 wt\% of GelNB (Figure 1A) was mixed with different weight contents of PEG4SH (Figure 1B) to give a range of thiol-norbornene ratios (i.e.,

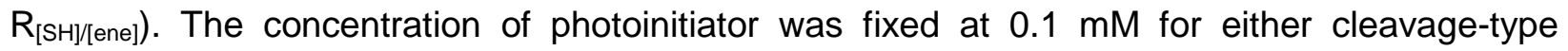


initiator LAP (Figure 1C) or non-cleavage type initiator EY (Figure 1D). Typically, non-cleavage (type II) photoinitiator eosin-Y was used with visible light-mediated crosslinking ${ }^{[11,25-27]}$, whereas cleavage-type (type I) photoinitiator LAP was used in conjunction with UV light-mediated crosslinking ${ }^{[10,28,29]}$ (Figure 1E). Figure 1F shows a comparison of the resulting hydrogel moduli between gelation initiated by visible light or UV light. Gels crosslinked via visible light

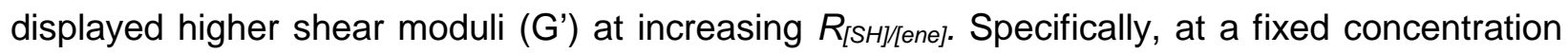
of bioactive component (i.e., 5 wt\% GelNB), gel moduli could be easily tuned from $\sim 2,200 \mathrm{~Pa}$ to $\sim 8,000 \mathrm{~Pa}$. Surprisingly, thiol-norbornene crosslinking initiated by UV light at $0.1 \mathrm{mM}$ LAP failed to gel at $R_{[S H][\text { ene] }}$ below unity. Even with a thiol-to-ene ratio of 1, LAP initiated gels were about 10-fold weaker than gels initiated via EY (G': 800 Pa vs. $\sim 8000 \mathrm{~Pa}$ ). These results suggest that it was possible to achieve orthogonal and visible light mediated photocrosslinking of GelNB hydrogels using low concentration of eosin-Y as the only photoinitiator. Furthermore, $0.1 \mathrm{mM}$ of LAP, a known UV light initiator, was insufficient in initiating GeINB-PEG4SH gelation.

\subsection{Effect of Initiator Type on Visible Light Mediated Crosslinking of GelNB Hydrogels}

LAP is routinely used as a UV-light photoinitiator owing to its high molar absorbability in the UV light wavelengths, high water solubility, and cytocompatibility. To better understand the excitability of both LAP and EY, we scanned the absorbance of both photoinitiators across UV and visible light wavelengths $(250-700 \mathrm{~nm}$, Figure $2 \mathrm{~A})$. We calculated the molar absorptivity of LAP at $365 \mathrm{~nm}$ to be around $218 \mathrm{M}^{-1} \mathrm{~cm}^{-1}$, a value consistent with the literature. ${ }^{[10]}$ It was also determined that LAP has limited molar absorptivity $\left(\sim 30 \mathrm{M}^{-1} \mathrm{~cm}^{-1}\right)$ between 400-405 nm, suggesting that it was possible to initiate thiol-norbornene gelation using visible light and LAP. Indeed, previous studies have exploited the weak excitability of LAP in the visible light range to initiate crosslinking of hydrogels, even though the concentration of LAP needed to achieve gelation was relatively high $(>6 \mathrm{mM}){ }^{[30]}$ On the other hand, EY has extremely high molar absorptivity at $525 \mathrm{~nm}\left(100,000 \mathrm{M}^{-1} \mathrm{~cm}^{-1}\right)$, indicating that visible light and EY-initiated gelation 
would be effective at low EY concentration. ${ }^{[7]}$ To establish the concentration of LAP needed to achieve a similar degree of hydrogel crosslinking to that using $0.1 \mathrm{mM}$ of EY under the same visible light exposure, we tested the gelation of $5 \mathrm{wt} \%$ GeINB and $2.8 \mathrm{wt} \% \mathrm{PEG} 4 \mathrm{SH}\left(R_{[S H] /[e n e]}=\right.$ 1). As shown in Figure 2B, the shear modulus ( $\left.G^{\prime}\right)$ of the hydrogel increased at increasing concentrations of LAP. Furthermore, gelation using $4 \mathrm{mM}$ of LAP achieved similar moduli to that obtained when using $0.1 \mathrm{mM}$ EY with the same macromer contents (Figure 1F compared with Figure 2B). We further examined visible light initiated gelation using 5 or 8 wt $\%$ of GelNB at $R_{[S H] /[e n e]}=1$ in the presence of either $4 \mathrm{mM} L A P$ or $0.1 \mathrm{mM} \mathrm{EY}$. As shown in Figure 2C, gels comprised of the same formulations displayed similar shear moduli between the two initiators. Together, we were able to establish orthogonally crosslinked gelatin hydrogels using either cleavage-type or non-cleavage type photoinitiator under the same visible light exposure. However, a 40-fold higher concentration of cleavage-type initiator (i.e., 4 mM LAP) was required to achieve a similar gelation efficiency to that using non-cleavage type initiator (i.e., $0.1 \mathrm{mM} \mathrm{EY}$ ).

While experiments in Figure 2 have established the concentration of LAP needed to achieve a similar degree of crosslinking when comparing to that initiated by EY, all gels were formed after 5 minutes of visible light exposure and it was unclear whether the two photoinitiators (i.e., $0.1 \mathrm{mM} E Y$ and $4 \mathrm{mM}$ LAP) exhibited the same initiation efficiency. One simple way to determine the effect of photoinitiator type on gelatin kinetics was to conduct in situ photorheometry. To this end, precursor solutions containing macromers GeINB and PEG4SH, as well as either $0.1 \mathrm{mM}$ EY or $4 \mathrm{mM}$ LAP were mixed and followed by visible light exposure (400-700 nm, 70k Lux). For both photoinitiators, storage or shear moduli (G') rapidly surpassed loss moduli (G") and reached similar values ( $2 \mathrm{kPa})$ after visible light was switched on for 300 seconds (Figure 3A, 3B). However, the gel point, an indication of how rapid a crosslinked network started to form, of GeINB-PEG4SH gelation using EY was nearly 1.7x faster than that using LAP (Figure 3C, 24 sec vs. 42 sec for EY and LAP, respectively). These results suggest that the high molar absorbability of EY contributed to faster thiol-norbornene gelation kinetics. 


\subsection{Proteolytic Degradation of GelNB Hydrogels}

Previously, we have shown that modularly crosslinked GelNB hydrogels could be degraded proteolytically by chymotrypsin, ${ }^{[5]}$ an endopeptidase that cleaves the C-terminus of any Phe, Trp, or Tyr residue unless immediately followed by Pro. Here, we investigated the proteolytic degradability of orthogonally crosslinked gelatin-based gels using collagenase, also named matrix metalloproteinase 1 (MMP-1), which cleaves sequences such as Gly-Pro-GInGly-Ile-Ala-Gly-GIn between Gly and Ile. ${ }^{[31-34]}$ The degradation of gelatin-based hydrogels in the presence of MMP-1 would demonstrate the ability of these matrices to be remodeled in the presence of cell-secreted proteases. To this end, hydrogels were formed by either $5 \mathrm{wt} \%$ or 8 wt $\%$ of GelNB with $R_{[S H][\text { ene] }}=1$, which resulted in gels with different crosslinking densities and initial moduli. Gel crosslinking density was also adjusted through using different values of $R_{[S H] /[e n e]}(0.4$ or 1 , by adjusting concentrations of PEG4SH). As shown earlier (Figure 2C), GeINB-PEG4SH hydrogels crosslinked by $0.1 \mathrm{mM}$ eosin-Y or $4 \mathrm{mM}$ LAP displayed a similar degree of crosslinking. Regardless of photoinitiator type, these hydrogels exhibited almost identical susceptibility to MMP-1 mediated proteolysis (Figure 4A). Specifically, all hydrogels with a lower degree of crosslinking (G' $-3 \mathrm{kPa}$ ) degraded rapidly following $10 \mathrm{U} / \mathrm{mL}$ of MMP-1 treatment, and the linearity of the mass loss results suggests a surface erosion mechanism. However, the degradation of hydrogels formed with a higher degree of crosslinking (G' $>8 \mathrm{kPa}$ and $15 \mathrm{kPa}$ ) required a much longer time frame to achieve gel erosion (Figure 4B). Nearly 30 hours into the study, gels still exhibited $70-80 \%$ of their original mass. This result was in accordance with our previous work where the degree of GeINB hydrogel crosslinking affects the mode of proteolytic degradation. ${ }^{[5]}$ More specifically, during the first 8 hours of MMP-1 treatment, gels exhibited an increase in mass (i.e., negative mass loss \%). During this period, gels also increased in volumetric size due to the partial cleavage of gelatin and higher amount of water intake. However, their structural integrity remained intact since these gels contained higher concentration of crosslinks. As more degradation happened, gels began to lose their 
structural integrity and the mass loss started to increase. Finally, hydrogels crosslinked by 8 wt\% GelNB (G' $15 \mathrm{kPa}$ ) exhibited higher mass gain in the first degradation phase but were more resistant to MMP-1 mediated degradation, as indicated by a lower \% mass loss when comparing to gels formed at 5 wt $\%$ GelNB. Nonetheless, all GelNB-PEG4SH hydrogels were susceptible to exogenously added MMP-1, an indication that these chemically crosslinked matrices could be degraded by cell-secreted proteases.

\subsection{Influence of Photoinitiator Type on Viability and Function of Encapsulated HepaRG Cells}

HepaRG cells were encapsulated and cultured in 5 wt\% GelNB-PEG4SH hydrogels for 28 days to evaluate the potential impact of 3D culture on hepatocytic differentiation within the gels. Hydrogels obtained from using either $0.1 \mathrm{mM}$ EY or $4 \mathrm{mM}$ LAP as the photoinitiator exhibited similar shear moduli ( $-3,000 \mathrm{~Pa}$, data not shown). Figure 5A shows the viability of the encapsulated cells at day-1, 14, and 28 post-encapsulation. These live/dead staining images reveal that both LAP and EY-initiated GeINB-PEG4SH hydrogels were highly cytocompatible for in situ encapsulation of HepaRG cells as only a limited number of dead cells (stained red) were observed in both systems one day post-encapsulation (Figure 5A, left panel). After 14 (Figure 5A middle panel) and 28 days (Figure 5A, right panel) of 3D culture, HepaRG cells formed multi-cell clusters with no visible dead cells, suggesting that these visible light polymerized hydrogels supported the growth of HepaRG cells in 3D. When comparing live/dead staining images captured at day-1 and day-14 post-encapsulation, cell density appeared to be much lower in the day-14 images. Furthermore, cells within EY gels seemingly exhibit slightly larger clusters than those within LAP gels. It was possible that some cells died sometime during the first two weeks of 3D culture. Most of these dead cells were not captured in the live/dead staining as the staining was only capable of capturing a 'snap-shot' in time of the cell viability at which staining was performed. In addition to qualitative live/dead staining, we also monitored 
cellular metabolic activity using AlamarBlue ${ }^{\circledR}$ assay over the course of the study. As shown in Figure 5B, regardless of the initiator type, cell metabolic activity dropped to almost half of the initial values during the first 2 weeks. The decrease in quantitative metabolic activity assay confirms the qualitative live/dead staining results and supports the notion that some cells died during the first two weeks of 3D culture. Interestingly, from day-14 to day-28, HepaRG cells encapsulated in EY-initiated gels exhibit slight increases in metabolic activity, whereas the metabolic activity remained relatively unchanged in cells encapsulated within LAP initiated hydrogels. Although appeared to be mostly alive in the two sets of hydrogels, cells encapsulated within the EY gels may proliferated slightly more than those in the LAP gels, thus contributing to higher metabolic activity occurred at later time points (i.e., day-14 and 28).

As hepatocyte progenitor cells, HepaRG cells are capable of being differentiated into hepatocytes. To gain understandings of the effect of photoinitiator type on HepaRG cell fate, we performed functional assay on CYP450 family, a class of enzymes responsible for detoxification and metabolism within hepatocytes. ${ }^{[35]}$ The enzyme activity was evaluated 14 and 28 days post encapsulation (Figure 5C) and no detectable levels of CYP450 activity was found at day-14 post-encapsulation. At day-28 post-encapsulation, however, significantly higher CYP450 enzymatic activity was detected in both sets of cell-laden hydrogels. As can be seen in Figure 5A, HepaRG cells formed small multi-cell clusters after 28 days of 3D culture and it was possible that the increase in cell-cell interactions promoted hepatocyte-specific function in HepaRG multi-cell clusters formed in 3D over the course of four weeks. In a previous study, it was reported that undifferentiated HepaRG cells did not exhibit detectable CYP enzymatic activity within 14 days of 2D culture. In our current study, HepaRG cells cultured in 3D hydrogels also did not express detectable CYP activity before day-14. It is worth noting that although CYP450 activity increased over time, there was no statistical significant difference between cells cultured in gels formed by EY or LAP initiated photopolymerization. 


\subsection{Potential Hepatocytic Differentiation of HepaRG Cells in DMSO-free 3D Culture}

Previous works have shown that HepaRG cells could be differentiated toward hepatocyte lineage using DMSO treatment. As the first step towards promoting hepatocytic differentiation of HepaRG cells using DMSO-free 3D cell culture, we investigated the mRNA expression level of two important hepatocyte-specific genes, hepatocyte nuclear factor $4 \alpha$ (HNF4a) and sodiumtaurocholate co-transporting polypeptide (NTCP), ${ }^{[14,36-38]}$ in cells cultured in EY or LAP initiated GeINB-PEG4SH hydrogels. HNF4a was chosen because it is a nuclear transcription factor that controls the expression of many hepatic genes, including the CYP450 family. On the other hand, NTCP was evaluated because it is a hepatic specific protein important for bile transport and the entry of hepatitis B virus (HBV), ${ }^{[39]}$ a virus that only infects human hepatocytes. HepaRG cells grown in 24-well tissue culture plates without (i.e., proliferation media for 28 days) or with DMSO (i.e., proliferation media for 14 days, followed by 1\% DMSO treatment for another 14 days) were used as controls. After 2 and 4 weeks of 2D or 3D culture, cells were collected for RNA isolation and gene expression analyses (Figure 6A). Figure 6B shows that the expressions of HNF4a in HepaRG cells cultured in 3D hydrogels were slightly higher but not statistically significant than that in the 2D control after 2 weeks. However, as seen in Figure 6C, the expressions of NTCP in 3D culture were significantly higher than the 2D control group. Specifically, EY and LAP initiated GeINB gels supported NTCP expression in HepaRG cells nearly 5 and 3 -fold higher when comparing with cells maintained on 2D tissue culture plastic ${ }^{\star \star \star} p<0.0001$ for both). Furthermore, the expression of NTCP in EY-initiated gels was significantly higher than that in LAP initiated gels ( $\left.{ }^{\star \star} p<0.001\right)$. Between day-14 and day-28, a group of 2D cultured cells were subjected to 1\% DMSO treatment to induce hepatocytic differentiation whereas cells cultured in 3D were still maintained in DMSO-free condition. The two-week DMSO treatment in cells cultured in 2D did not yield significant changes in HNF4a and NTCP mRNA levels (Figures 6B, C). The expression of both HNF4 $\alpha$ and NTCP in HepaRG cells cultured in LAP initiated gels were also similar to that found in 2D culture. Interestingly, 
both HNF4a and NTCP levels in cells cultured in EY-initiated gels were significantly higher than that in 2D and in 3D LAP initiated gels (** $p<0.001 ;{ }^{\star} p<0.05$ respectively). HNF4 $\alpha$ and NTCP) on the mRNA levels. These results are consistent with our findings that NTCP expression in hepatocellular carcinoma cells (e.g., HepG2 and Huh7) was upregulated using 3D hydrogel culture, ${ }^{[35]}$ suggesting that a 3D platform has positive effects on hepatocyte differentiation. It is likely that the 3D hydrogel environment not only promoted crucial cell-cell interactions, but also provided cell-matrix interactions that were otherwise deprived off in conventional 2D TCP culture. As a result, cell differentiation was positively influenced when they were cultured within 3D biomimetic matrices.

As discussed previously, EY has a higher molar absorptivity within the visible light range as compared with LAP. As such, it may also produce a greater number of reactive oxygen species during the polymerization process that may induce greater amounts of stress on cells. A recent study has suggested that the expression and secretion of Augmenter of Liver Regeneration (ALR), a factor capable of protecting hepatocytes against stressors, increased significantly when the cells encounter environmental stress. ${ }^{[40]}$ ALR could promote hepatocyte viability and liver regeneration through increasing hepatocyte proliferation. ${ }^{[40,41]}$ Recent work has also shown that upon the deletion of this protein, hepatocytes lost their normal functions resulting in the formation of hepatocellular carcinoma in mice. ${ }^{[42]}$ Therefore, it may be likely that ALR expression is elevated in the cells encapsulated in EY gels due to the potential increase of stress within these particular hydrogels. While future work is required to characterize the differentiation of HepaRG in 3D culture, the encouraging results reported herein have demonstrated the suitability of the visible light crosslinked gelatin based hydrogels for 3D differentiation of hepatic progenitor cells. 


\section{Conclusions}

This work systematically compared the effects of photoinitiator type on hydrogel formation and cytocompatibility, as well as their suitability to serve as an in vitro culture system for culturing hepatic progenitor cells in 3D. Although chemically crosslinked gelatin hydrogels could be initiated by either cleavage-type or non-cleavage type photoinitiator, a high concentration of cleavage-type initiator was required to achieve similar gelation efficiency. We also demonstrated that modular GelNB-PEG4SH hydrogels crosslinked by both types of initiators (e.g., $0.1 \mathrm{mM}$ eosin-Y or $4 \mathrm{mM}$ LAP) displayed similar gelation/degradation profiles. In addition, GelNB hydrogels possess comparable cytocompatibility for hepatic progenitor cells HepaRG. Finally, EY-initiated gelation promoted higher expression of hepatic-specific genes (e.g., HNF4a and NTCP), indicative of potential hepatic differentiation in HepaRG cells free of DMSO treatment.

Acknowledgements: This work was supported in part by a Research Support Funds Grant (RSFG) from the IUPUI Office of the Vice Chancellor for Research (OVCR) and an Early Faculty Career Development (CAREER) award from the National Science Foundation (NSF, DMR\#1452390).

Keywords: Poly(ethylene glycol), gelatin, thiol-ene hydrogel, photopolymerization, photoinitiator, HepaRG 


\section{References}

1. Benton, J.A., et al., Photocrosslinking of gelatin macromers to synthesize porous hydrogels that promote valvular interstitial cell function. Tissue Eng Part A, 2009. 15(11): p. 3221-30.

2. Yue, K., et al., Synthesis, properties, and biomedical applications of gelatin methacryloyl (GelMA) hydrogels. Biomaterials, 2015. 73: p. 254-71.

3. $\mathrm{Xu}, \mathrm{K}$., et al., Thiol-ene Michael-type formation of gelatin/poly(ethylene glycol) biomatrices for three-dimensional mesenchymal stromal/stem cell administration to cutaneous wounds. Acta Biomater, 2013. 9(11): p. 8802-14.

4. Munoz, Z., H. Shih, and C.-C. Lin, Gelatin hydrogels formed by orthogonal thiolnorbornene photochemistry for cell encapsulation. Biomaterials Science, 2014. 2(8): p. 1063-1072.

5. Greene, T. and C.-C. Lin, Modular Cross-Linking of Gelatin-Based Thiol-Norbornene Hydrogels for in Vitro 3D Culture of Hepatocellular Carcinoma Cells. ACS Biomaterials Science \& Engineering, 2015. 1: p. 1314-1323.

6. Sridhar, B.V., et al., A Biosynthetic Scaffold that Facilitates Chondrocyte-Mediated Degradation and Promotes Articular Cartilage Extracellular Matrix Deposition. Regen Eng Transl Med, 2015. 1(1-4): p. 11-21.

7. Shih, H. and C.C. Lin, Visible-light-mediated thiol-ene hydrogelation using eosin-Y as the only photoinitiator. Macromol Rapid Commun, 2013. 34(3): p. 269-73.

8. Mazaki, T., et al., A novel, visible light-induced, rapidly cross-linkable gelatin scaffold for osteochondral tissue engineering. Sci Rep, 2014. 4: p. 4457.

9. Ding, Y., et al., Photo-cross-linking approach to engineering small tyrosine-containing peptide hydrogels with enhanced mechanical stability. Langmuir, 2013. 29(43): p. 13299-306.

10. Fairbanks, B.D., et al., Photoinitiated polymerization of PEG-diacrylate with lithium phenyl-2,4,6-trimethylbenzoylphosphinate: polymerization rate and cytocompatibility. Biomaterials, 2009. 30(35): p. 6702-7.

11. Shih, H., R.G. Mirmira, and C.C. Lin, Visible light-initiated interfacial thiol-norbornene photopolymerization for forming islet surface conformal coating. J Mater Chem B Mater Biol Med, 2015. 3: p. 170-175.

12. Gripon, P., et al., Infection of a human hepatoma cell line by hepatitis $B$ virus. Proc Natl Acad Sci U S A, 2002. 99(24): p. 15655-60.

13. Aninat, C., et al., Expression of cytochromes P450, conjugating enzymes and nuclear receptors in human hepatoma HepaRG cells. Drug Metab Dispos, 2006. 34(1): p. 75-83.

14. Cerec, V., et al., Transdifferentiation of hepatocyte-like cells from the human hepatoma HepaRG cell line through bipotent progenitor. Hepatology, 2007. 45(4): p. 957-67.

15. Lubberstedt, M., et al., HepaRG human hepatic cell line utility as a surrogate for primary human hepatocytes in drug metabolism assessment in vitro. J Pharmacol Toxicol Methods, 2011. 63(1): p. 59-68. 
16. Malinen, M.M., et al., Differentiation of liver progenitor cell line to functional organotypic cultures in 3D nanofibrillar cellulose and hyaluronan-gelatin hydrogels. Biomaterials, 2014. 35(19): p. 5110-21.

17. Higuchi, Y., et al., Functional polymer-dependent $3 D$ culture accelerates the differentiation of HepaRG cells into mature hepatocytes. Hepatol Res, 2016.

18. Guguen-Guillouzo, C. and A. Guillouzo, General review on in vitro hepatocyte models and their applications. Methods Mol Biol, 2010. 640: p. 1-40.

19. Rebelo, S.P., et al., HepaRG microencapsulated spheroids in DMSO-free culture: novel culturing approaches for enhanced xenobiotic and biosynthetic metabolism. Arch Toxicol, 2015. 89(8): p. 1347-58.

20. Hoekstra, R., et al., Phase 1 and phase 2 drug metabolism and bile acid production of HepaRG cells in a bioartificial liver in absence of dimethyl sulfoxide. Drug Metab Dispos, 2013. 41(3): p. 562-7.

21. Lau, T.T., et al., Formation of model hepatocellular aggregates in a hydrogel scaffold using degradable genipin crosslinked gelatin microspheres as cell carriers. Biomed Mater, 2012. 7(6): p. 065003.

22. Gevaert, E., et al., Galactose-functionalized gelatin hydrogels improve the functionality of encapsulated HepG2 cells. Macromol Biosci, 2014. 14(3): p. 419-27.

23. Raza, A., C.S. Ki, and C.C. Lin, The influence of matrix properties on growth and morphogenesis of human pancreatic ductal epithelial cells in 3D. Biomaterials, 2013. 34(21): p. 5117-27.

24. Lin, T.Y., J.C. Bragg, and C.C. Lin, Designing Visible Light-Cured Thiol-Acrylate Hydrogels for Studying the HIPPO Pathway Activation in Hepatocellular Carcinoma Cells. Macromol Biosci, 2016. 16(4): p. 496-507.

25. Lin, C.C., C.S. Ki, and H. Shih, Thiol-norbornene photo-click hydrogels for tissue engineering applications. J Appl Polym Sci, 2015. 132(8).

26. Fisher, J.P., et al., Photoinitiated Polymerization of Biomaterials. Annual Review of Materials Research, 2001. 31(1): p. 171-181.

27. Cruise, G.M., et al., A sensitivity study of the key parameters in the interfacial photopolymerization of poly(ethylene glycol) diacrylate upon porcine islets. Biotechnol Bioeng, 1998. 57(6): p. 655-65.

28. Shih, H. and C.C. Lin, Photoclick Hydrogels Prepared from Functionalized Cyclodextrin and Poly(ethylene glycol) for Drug Delivery and in Situ Cell Encapsulation.

Biomacromolecules, 2015. 16(7): p. 1915-23.

29. Ki, C.S., et al., Thiol-ene hydrogels as desmoplasia-mimetic matrices for modeling pancreatic cancer cell growth, invasion, and drug resistance. Biomaterials, 2014. 35(36): p. 9668-77.

30. Caliari, S.R., et al., Stiffening hydrogels for investigating the dynamics of hepatic stellate cell mechanotransduction during myofibroblast activation. Sci Rep, 2016. 6: p. 21387.

31. Kishen, A., et al., Characterizing the collagen stabilizing effect of crosslinked chitosan nanoparticles against collagenase degradation. Dent Mater, 2016.

32. Chung, L., et al., Collagenase unwinds triple-helical collagen prior to peptide bond hydrolysis. EMBO J, 2004. 23(15): p. 3020-30. 
33. Birkedal-Hansen, H., et al., Matrix metalloproteinases: a review. Crit Rev Oral Biol Med, 1993. 4(2): p. 197-250.

34. Arnold, L.H., et al., The interface between catalytic and hemopexin domains in matrix metalloproteinase-1 conceals a collagen binding exosite. J Biol Chem, 2011. 286(52): p. 45073-82.

35. Lin, T.Y., C.S. Ki, and C.C. Lin, Manipulating hepatocellular carcinoma cell fate in orthogonally cross-linked hydrogels. Biomaterials, 2014. 35(25): p. 6898-906.

36. Dianat, N., et al., Generation of functional cholangiocyte-like cells from human pluripotent stem cells and HepaRG cells. Hepatology, 2014. 60(2): p. 700-14.

37. Qiu, X., et al., Disruption of BSEP Function in HepaRG Cells Alters Bile Acid Disposition and Is a Susceptive Factor to Drug-Induced Cholestatic Injury. Mol Pharm, 2016. 13(4): p. 1206-16.

38. Stieger, B., The role of the sodium-taurocholate cotransporting polypeptide (NTCP) and of the bile salt export pump (BSEP) in physiology and pathophysiology of bile formation. Handb Exp Pharmacol, 2011(201): p. 205-59.

39. Yan, H., et al., Sodium taurocholate cotransporting polypeptide is a functional receptor for human hepatitis B and D virus. Elife, 2012. 1: p. e00049.

40. Vodovotz, Y., et al., Augmenter of liver regeneration (ALR) is a novel biomarker of hepatocellular stress/inflammation: in vitro, in vivo and in silico studies. Mol Med, 2012. 18: p. 1421-9.

41. Polimeno, L., et al., Augmenter of liver regeneration, a protective factor against ROSinduced oxidative damage in muscle tissue of mitochondrial myopathy affected patients. Int J Biochem Cell Biol, 2013. 45(11): p. 2410-9.

42. Gandhi, C.R., et al., Liver-specific deletion of augmenter of liver regeneration accelerates development of steatohepatitis and hepatocellular carcinoma in mice. Gastroenterology, 2015. 148(2): p. 379-391 e4. 


\section{Figures}
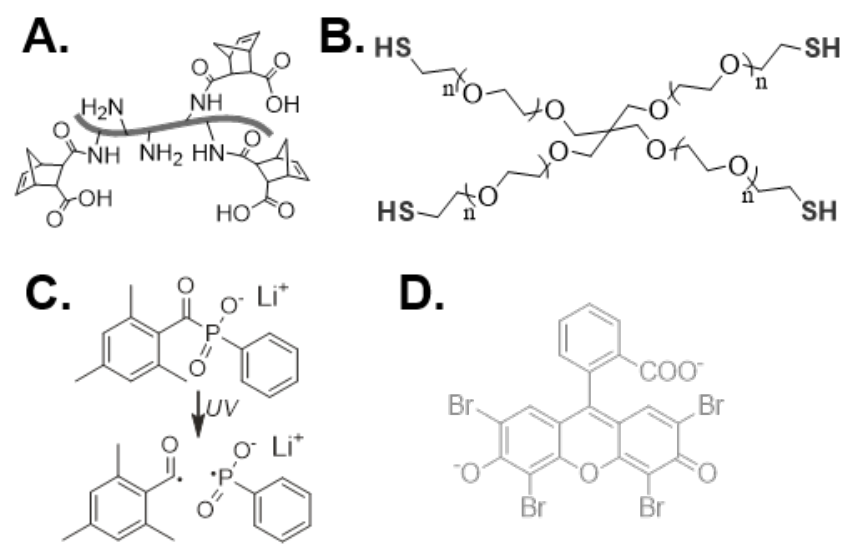

E.
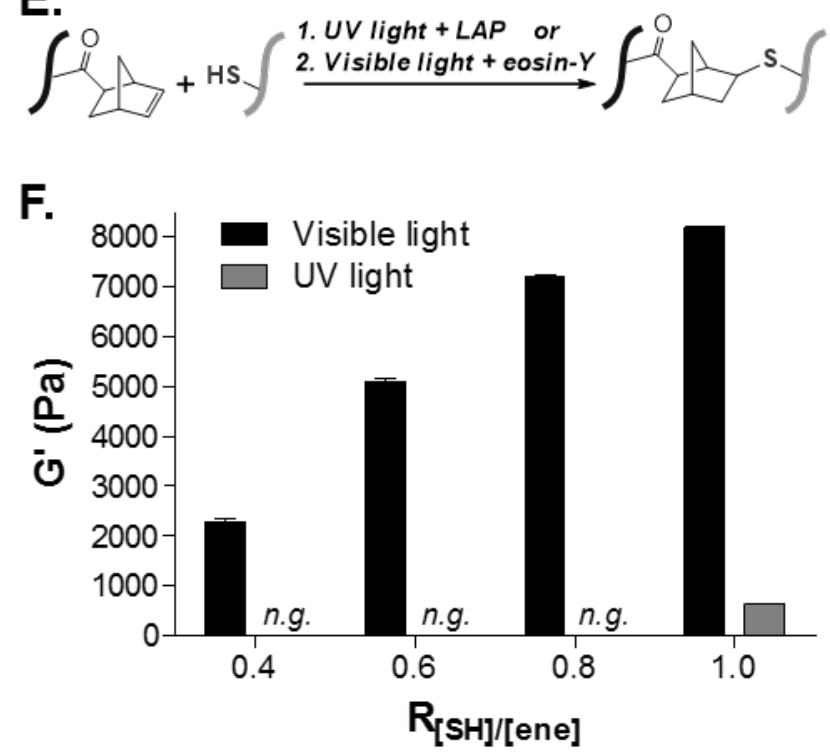

Figure 1. Chemical structures of: (A) norbornene-functionalized gelatin (GeINB), (B) PEG-tetrathiol (PEG4SH), (C) cleavage type photointiator lithium acylphosphinate (LAP), and (D) noncleavage type photointiator eosin-Y (EY). (E) Schematic of thiol-norbornene photoclick reaction using two initiation systems: 1. UV light + LAP and 2. Visible light + eosin-Y. (F) Shear moduli of 5 wt\% GelNB (i.e. $11.1 \mathrm{mM}$ norbornene) hydrogels crosslinked by PEG4SH at different thiol-toene molar ratios $\left(R_{[S H][[e n e]}\right)$. Gelation was initiated via either visible light $+0.1 \mathrm{mM} E Y$ or UV light + 0.1 mM LAP (n.g.: no gel). 


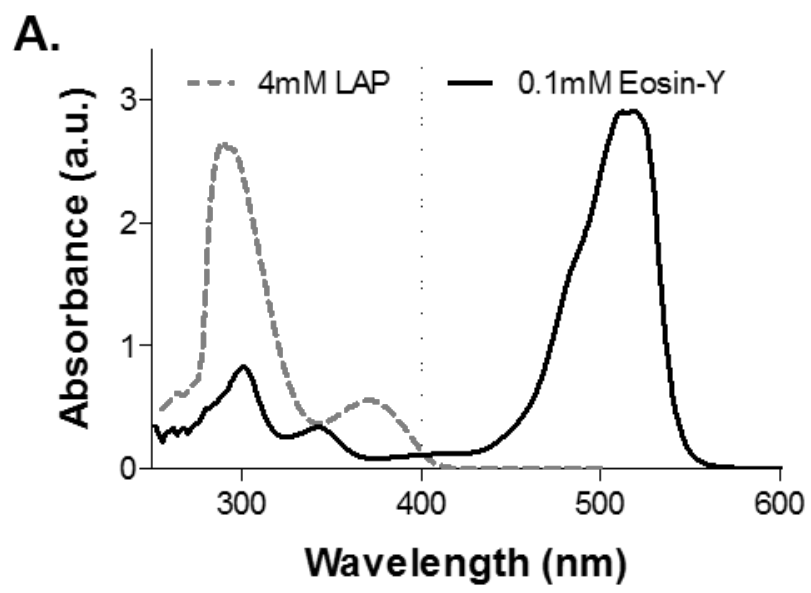

B.

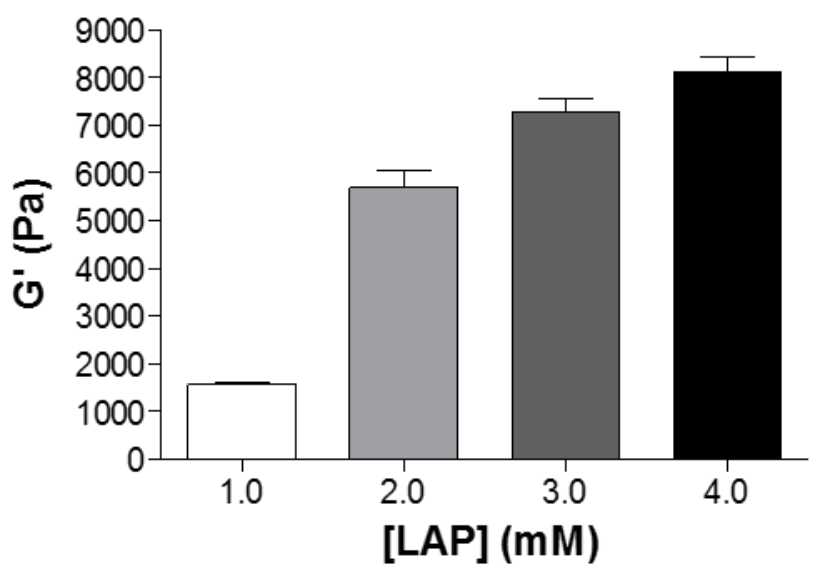

C.

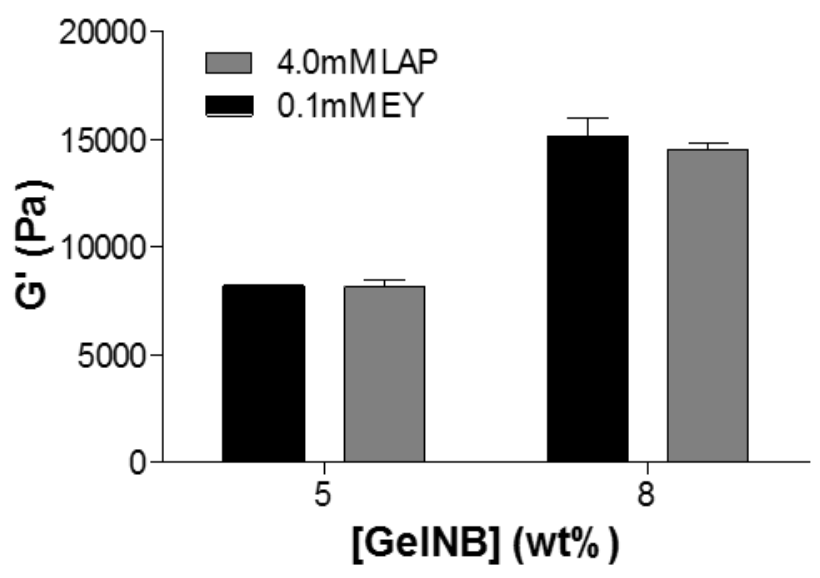

Figure 2. (A) UV/vis spectra of LAP/eosin-Y. (B) Shear moduli of 5 wt\% GelNB hydrogel crosslinked by 2.8 wt\% PEG4SH using different concentrations of LAP. (C) Shear moduli of 5 $w t \%$ and $8 w t \%(R=1.0)$ GelNB hydrogels initiated by LAP or EY. 

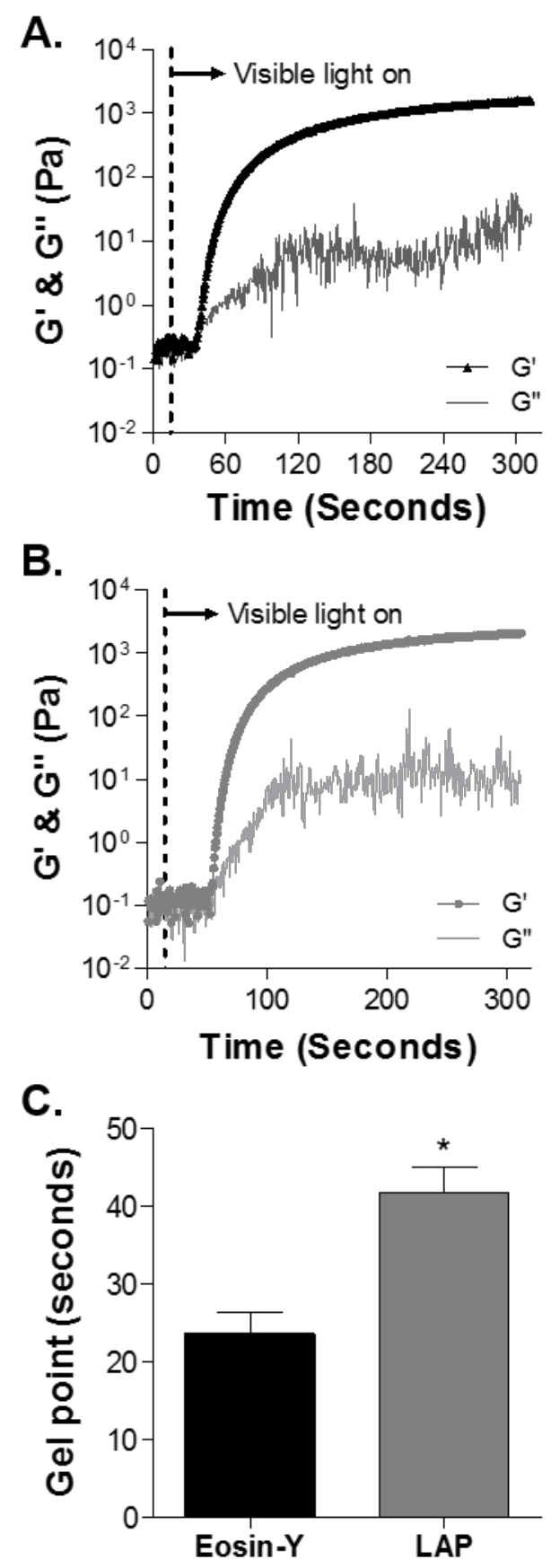

Figure 3. In situ photorheometry of GeINB-PEG4SH hydrogel crosslinking using (A) $0.1 \mathrm{mM}$ eosin-Y or (B) 4 mM LAP as the photoinitiator. Light was turned on 10 seconds after initiating the measurements. (C) Gel points of the two systems ( $\left.{ }^{\star} p<0.05\right)$. 

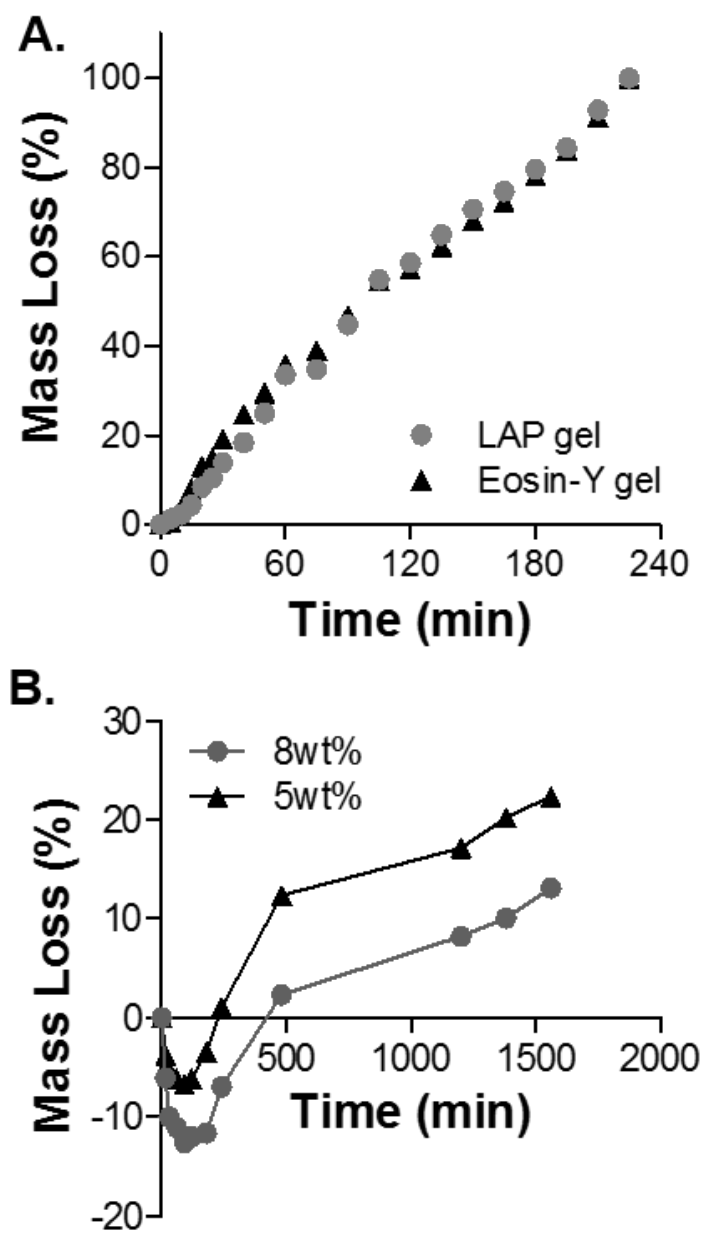

Figure 3. Collagenase I mediated $(10 \mathrm{U} / \mathrm{mL})$ proteolysis and mass loss of GelNB-A hydrogels cross-linked at (A.) similar densities (5wt\% GelNB R=0.4, $\sim 3000 \mathrm{~Pa}$ ) using both intiators LAP and EY and (B.) different GelNB content ( 5 or $8 w t \%$ GeINB R=1.0, $\sim 8000 \mathrm{~Pa}$ and $\sim 15000 \mathrm{~Pa}$ respectiviely) using only EY. 
A.
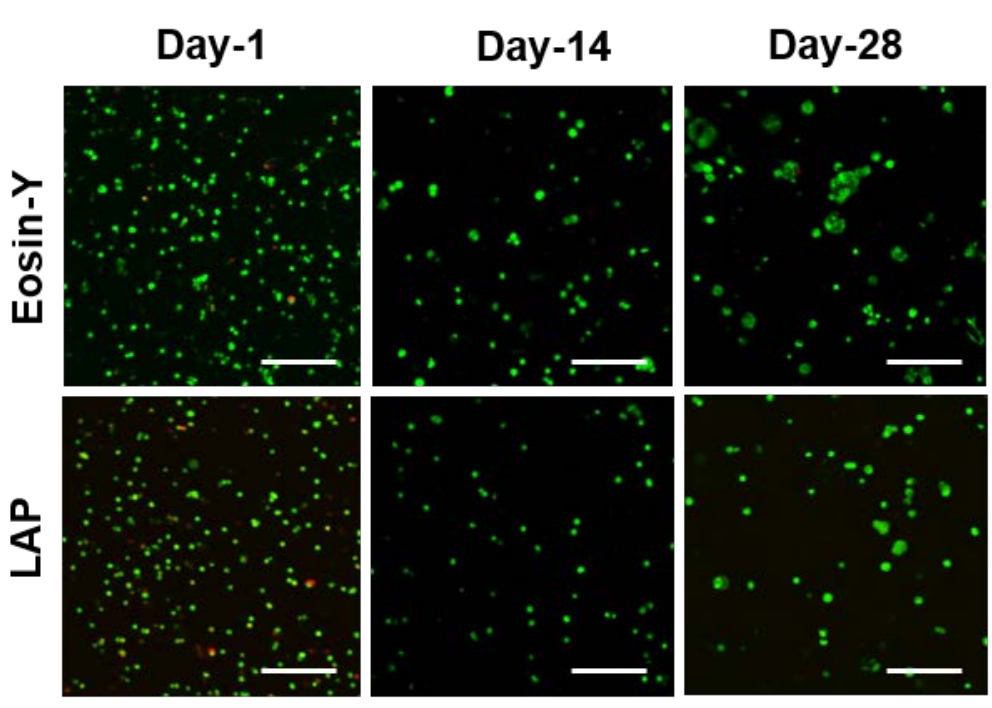

B.

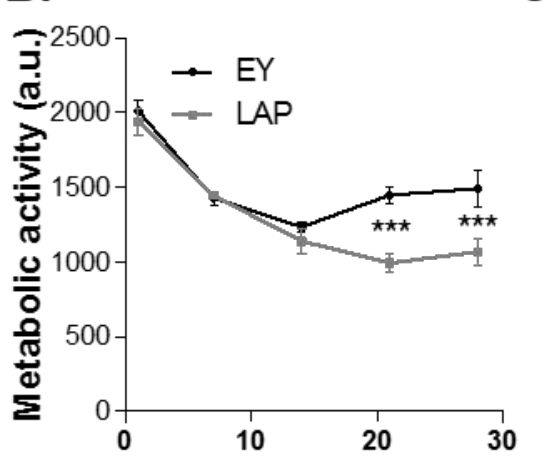

C.

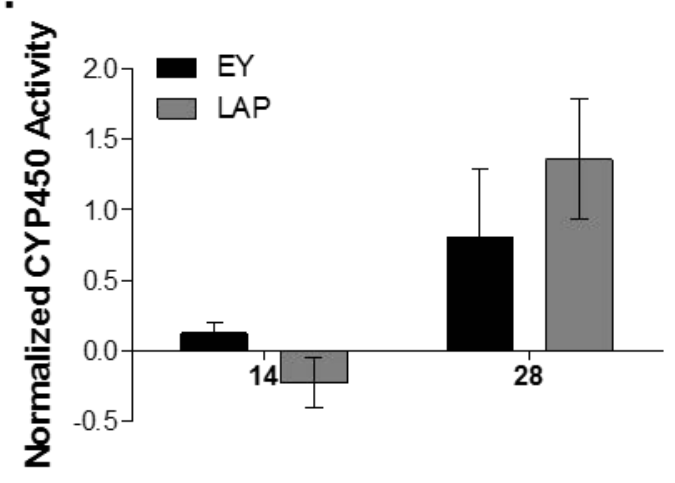

Figure 5. Effect of photointiator type on HepRG cell viability. (A) Representative live/dead staining and confocal Z-stack images of encapsulated HepaRG cells on days 1, 14, and 28. (B) Metabolic activity of HepaRG cells as a function of time. $\left({ }^{\star \star *} p<0.0001\right)$. (C) Normalized CYP450 enzymatic activity on days 14 and 28 post-encapsulation. CYP450 readings were normalized to cell metabolic activity from repective samples. 
A. 2D TCP plating

$10^{5}$ cells/well

$\left(5.3 \times 10^{4} / \mathrm{cm}^{2}\right)$

Evaluation

Begin DMSO treatment

Evaluation

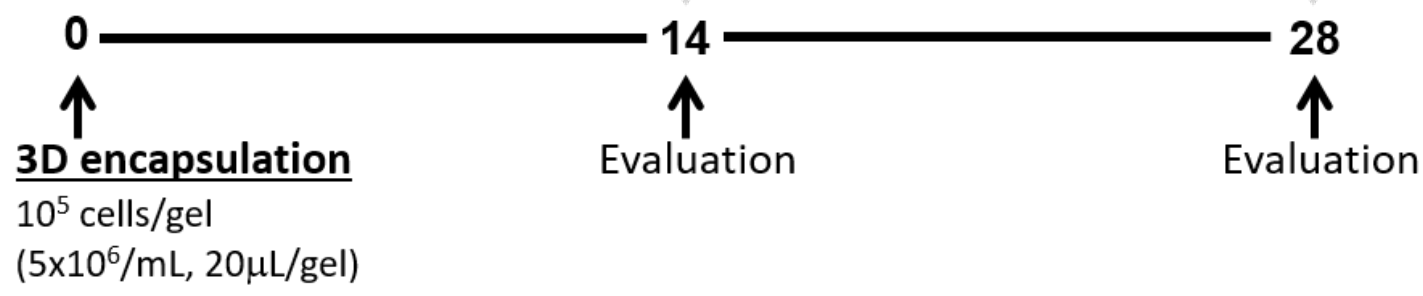

B.

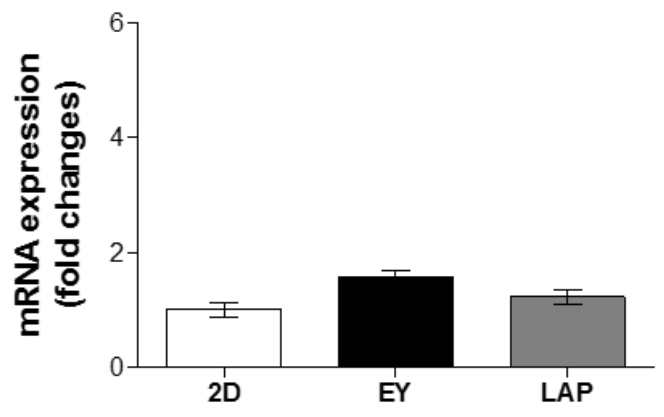

D.

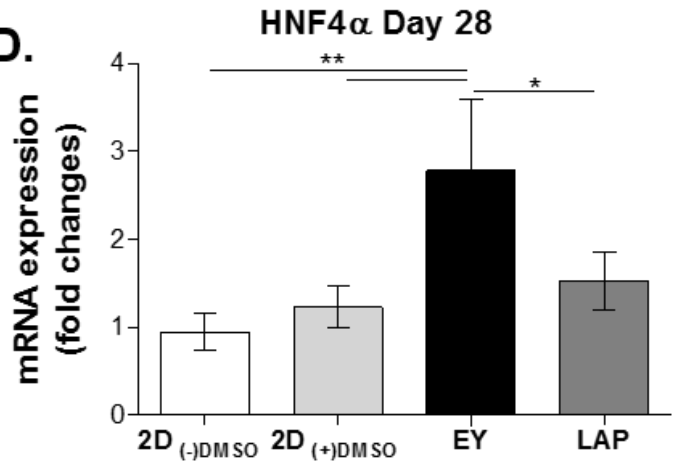

C.

NTCP Day 14

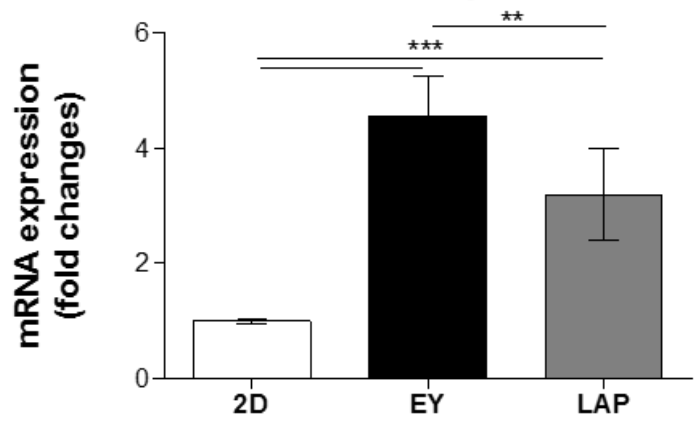

E.

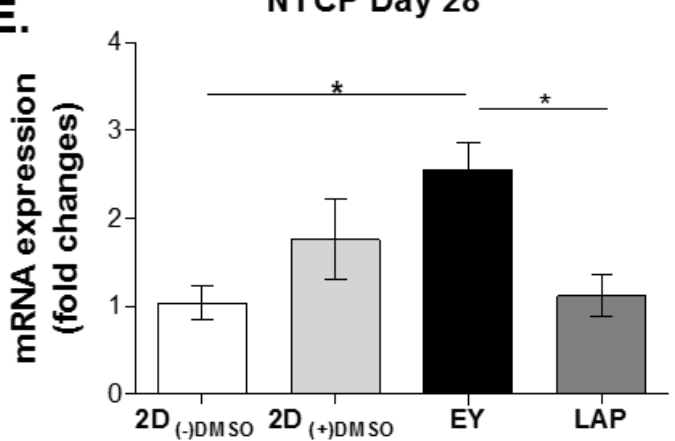

Figure 6. Effect of photointiator type on hepatocyte-specific gene expression. (A) RNA was isolated for quantitative real-time PCR evaluation on day 14 and day 28. (B, D) mRNA levels of hepatocyte nuclear factor 4 alpha (HNF4a) on day 14 (B) and day 28 (D). (C, E) mRNA levels of $\mathrm{Na}^{+}$taurocholate co-transporting polypeptide (NTCP) on day $14(\mathrm{C})$ and day $28(\mathrm{E})(\mathrm{n}=3$, mean \pm SEM. ${ }^{*} p<0.05 ;{ }^{*} p<0.01 ;{ }^{* \star} p<0.0001$. 1-fold: Gene expression level in cells cultured on 2D TCP on respective days). 
The table of contents entry

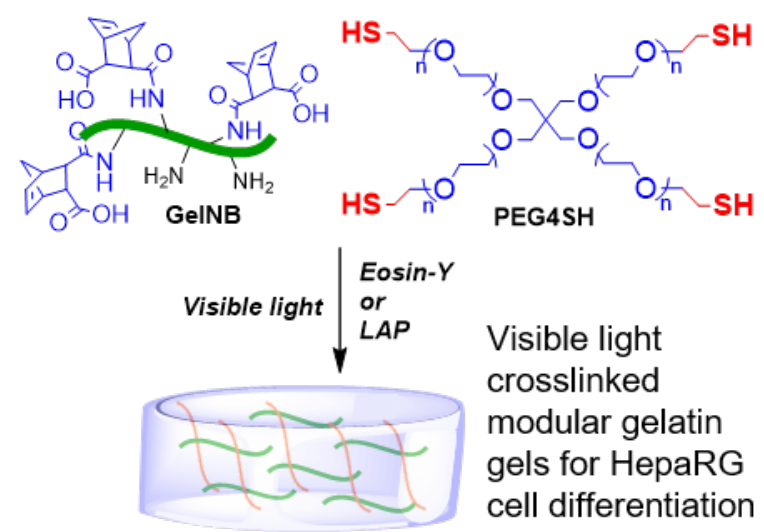

Gelatin-norbornene can be chemically crosslinked into 3D hydrogels through visible lightmediated thiol-norbornene photopolymerization with either type I or II photointiator. Both types of hydrogels have similar material properties and exhibit similar cytocompatibility for in situ encapsulation and 3D growth of liver progenitor cells HepaRG.

T. Greene, T.-Y. Lin, O.M. Andrisani, C.-C. Lin*

A comparative study of visible light polymerized gelatin hydrogels for 3D culture of hepatic progenitor cells. 